\title{
Measurement of Wire Deflection on Loading may Indicate Union in Ilizarov Constructs: A Pilot Study
}

\author{
Beth Lineham ${ }^{1}$, Todd Stewart ${ }^{2}$, John Ward ${ }^{3}$, Paul Harwood ${ }^{4}$
}

\section{Abstract}

Introduction: No entirely reliable method to assess union during llizarov treatment exists. Premature frame removal results in treatment failure, and alternative methods of assessment warrant investigation. Wire deflection might provide an indication of fracture site deformation on weight-bearing, indicating progress towards union. A previous in vitro study from our group demonstrated this approach may be clinically applicable. We investigated translation of this method into clinical practice in an observational pilot study.

Materials and methods: Patients with tibial shaft fractures treated with llizarov frames were recruited. A prototype depth gauge was used to measure wire deflection on weight-bearing. Investigators undertaking the measurement were blinded to the clinical stage of treatment, and clinicians caring for the patient were blinded to deflection results. Patient records were reviewed at the end of treatment to determine likely fracture stability at each time point. Deflection per $\mathrm{kg}$ of weight applied, per $\mathrm{mm}$ from the ring was compared between stable and unstable situations.

Results: Thirty-one measurements were obtained in 14 patients. The situation was deemed stable at 13 and unstable at 18 measurements. The median deflection in the stable group was 0.030 microns $/ \mathrm{kg} / \mathrm{mm}$ (IQR $0.005-0.104)$ and 0.165 microns $/ \mathrm{kg} / \mathrm{mm}$ (IQR 0.072-0.328) in the unstable group. This difference was statistically significant (Wilcoxon Mann-Whitney test $p=0.0014$ ). ROC curve analysis revealed that wire deflection was able to predict clinical stability (AUC $0.84, p<0.0001$ ). Various technical problems were encountered when using the device which would potentially limit its clinical utility in its current form.

Conclusion: In this set of observations, wire deflection was significantly associated with clinically and radiologically determined stability. Though various practical limitations were encountered in using the prototype measurement device, this proof-of-concept study supports further development of this approach. The research group plan to develop a smaller, more reliable device for further clinical testing in a larger group of patients.

Keywords: Frame, Ilizarov, Observational study, Pilot, Union, Wire deflection.

Strategies in Trauma and Limb Reconstruction (2021): 10.5005/jp-journals-10080-1537
\end{abstract}

\section{INTRODUCTION}

Ilizarov frame constructs are increasingly used for the treatment of lower limb trauma and deformity correction. There is currently no entirely reliable method of assessing bone union, and therefore, timing of frame removal can be difficult.' ${ }^{1}$ Current practice is to use clinical findings and medical imaging. However, the presence of the frame, particularly in complex fracture patterns, can make radiology difficult to interpret. Pain from fixation elements may be difficult to differentiate from fracture site pain, and patients may underplay their symptoms due to a desire for frame removal. Determining union in this way is subjective and has shown the potential to be inaccurate. ${ }^{2,3}$ Both premature and delayed frame removal can cause problems for the patient. Prolonged time in a frame can lead to delayed rehabilitation, with stiffness of adjacent joints, as well increased risk of pin site infection and other complications. ${ }^{4}$ Furthermore, patient experience is adversely affected, as frames can be difficult to live with, impairing return to work and social activities. This leads to increased indirect and direct treatment costs with loss of working time and an excess of clinic appointments and investigations. However, premature frame removal can result in treatment failure and complications such as non- or malunion. It is therefore pertinent to explore alternate methods to aid decision-making regarding progress to union during llizarov treatment.

Multiple alternative methods of assessing bone union have been investigated. Ultrasound can detect increasing consolidation at early

\begin{abstract}
1,4 Department of Trauma and Orthopaedics, School of Medicine, University of Leeds, Leeds, United Kingdom

${ }^{2}$ Department of Mechanical Engineering, University of Leeds, Leeds, United Kingdom

${ }^{3}$ School of Medicine, University of Leeds, Leeds, United Kingdom

Corresponding Author: Beth Lineham, Department of Trauma and Orthopaedics, School of Medicine, University of Leeds, Leeds, United Kingdom, e-mail: mn13bl@leeds.ac.uk

How to cite this article: Lineham B, Stewart T, Ward J, et al. Measurement of Wire Deflection on Loading may Indicate Union in Ilizarov Constructs: A Pilot Study. Strategies Trauma Limb Reconstr 2021;16(3):132-137.
\end{abstract}

Source of support: Nil

Conflict of interest: None

stages, but is unable to quantify bone in regenerate and therefore determine cortication or medullary differentiation. ${ }^{5-7}$ Vibration across the fracture site has also been described as a potential method, but interference from the external fixator construct has been shown to cause errors. ${ }^{2}$ Mechanical assessment of bone union in external fixators has been used in pre-clinical trials to some effect, ${ }^{6,8,9}$ and increasing fracture site stiffness should result in increased intrinsic force transfer and decreased force through the frame. However, no mechanical method is in current clinical use as previously described

(c) The Author(s). 2021 Open Access This article is distributed under the terms of the Creative Commons Attribution-Non Commercial-share alike license (https://creativecommons.org/licenses/by-nc-sa/4.0/) which permits unrestricted distribution, and non-commercial reproduction in any medium, provided you give appropriate credit to the original author(s) and the source, provide a link to the Creative Commons license, and indicate if changes were made. If you remix, transform, or build upon the material, you must distribute your contributions under the same license as original. The Creative Commons Public Domain Dedication waiver (http://creativecommons.org/publicdomain/zero/1.0/) applies to the data made available in this article, unless otherwise stated. 
techniques have been expensive and too time-consuming to be practical. We propose that deflection of the fine wires of the frame may show progress to union as with increasing union load transfer to the wires will be reduced and therefore deflection decreased. ${ }^{10}$ This method has previously been tested in a simulated setting and was able to distinguish between stable and unstable simulated bone frame constructs. ${ }^{11}$ It remains unclear whether this method might be translatable to clinical practice.

A clinical pilot study was therefore undertaken to determine whether a device for the measurement of wire deflection could reliably differentiate between mechanically stable and unstable clinical situations.

\section{Methods}

Institutional review board ethical approval for the study was granted.

\section{Patients}

Adult patients (aged 16 years or older) attending clinic with an llizarov frame in situ for a tibial fracture or tibial deformity correction were approached by a non-clinical member of the research team. Those indicating that they would like to take part in the study were counselled, and informed consent was obtained. Patients who were unable to give informed consent, were felt unable to comply with the instructions for the study or with inadequate mobility to at least partially weight-bear on the affected leg were excluded. Patients were included regardless of treatment stage.

\section{Wire Deflection Measurements}

A custom-made 316 Stainless Steel bracket was used to attach a depth gauge (Mitutoyo Digital Indicator, Mitutoyo, Andover, UK) to the first ring proximal to the fracture or osteotomy site. A custom lever arm was attached to the depth gauge to allow measurement within the ring (Fig. 1). Measurements were taken at a point as close to the bone as possible, and the position of measurement was recorded. Patients were then asked to weight-bear as tolerated on the affected leg whilst standing on a clinical weighing scale. A reading of maximal deflection was taken from the depth gauge and maximal weight transfer through the limb from the weighing scale. Measurements were repeated three times at each clinic visit. Measurements were taken by investigators not involved with the clinical care of the patients, and the clinicians delivering treatment were not made aware of the results.

\section{Assessment of Clinical Stability and Comparison with Wire Deflection Measurements}

Following completion of treatment, patient records and imaging were reviewed by the senior author. An assessment of likely clinical stability at the time of measurement was made. This was defined as unstable (fracture not united), indeterminate (fracture partially united) and stable (fracture united). The clinician was blinded to the deflection results when making this assessment. The clinician accessed the entire clinical record and was therefore able to include information obtained after the time the measurements were taken, improving the accuracy of their estimation. For subgroup analysis, given the smaller numbers of patient involved, observations classified as indeterminate were reassigned to the stable or unstable groups using the same criteria as above.

\section{Data Analysis}

Mean wire deflection per kg of weight applied per $\mathrm{mm}$ from the ring was determined for each patient at each clinic appointment from the three measurements taken. These results were compared between situation determined as stable and unstable. The distribution of wire deflection data was examined and found to be significantly skewed and did not meet assumptions for parametric analysis. Results are therefore summarised using the median and interquartile range. Deflection between groups was compared using a Wilcoxon Mann-Whitney (WMW) or a Kruskal-Wallis

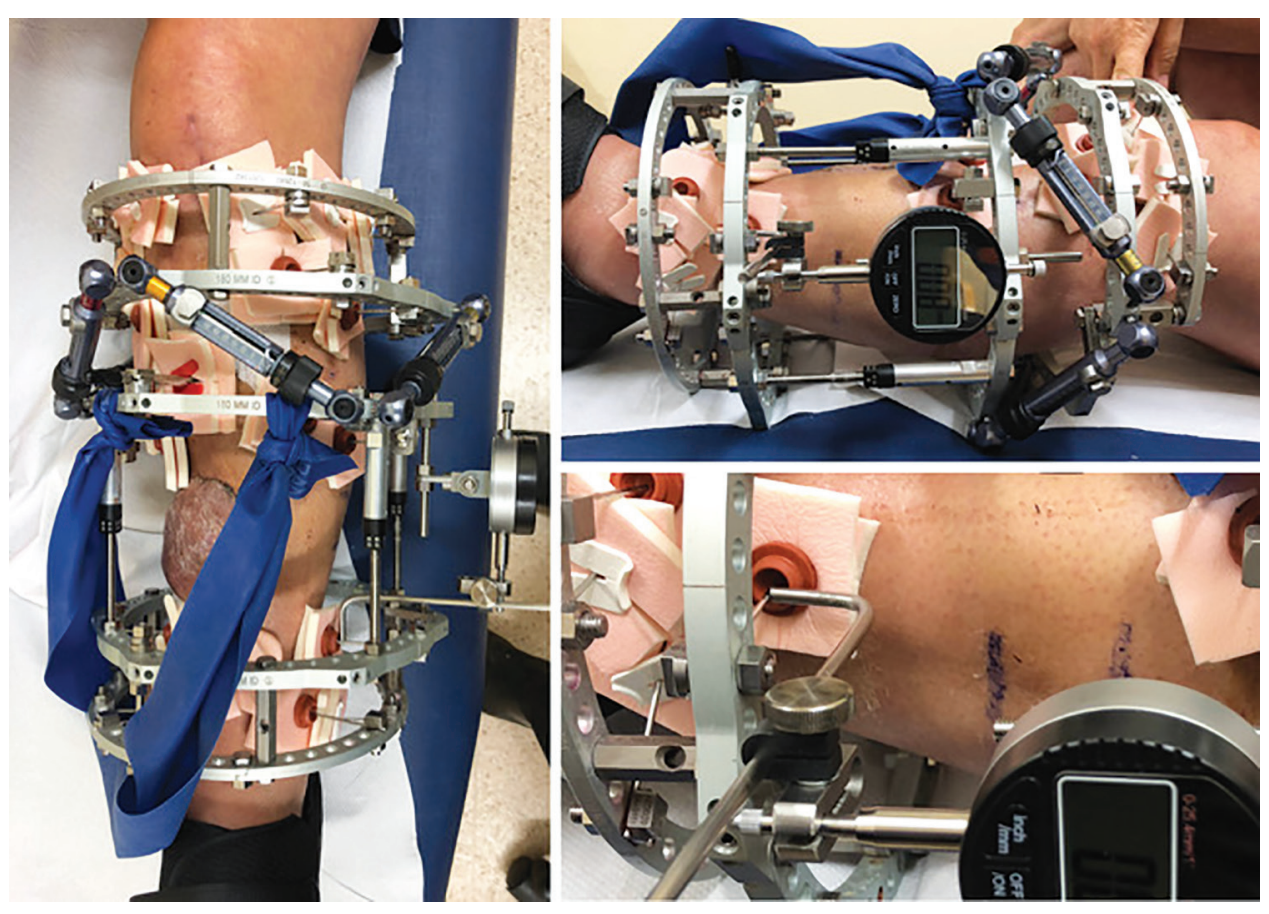

Fig. 1: Clinical photographs showing wire deflection measurement device in use on a hexapod frame 
(test) as appropriate. Subgroup analysis was undertaken using the Steel-Dwass-Critchlow-Fligner (SDCF) method of all-pairs comparison controlling for multiple testing. Receiver-operator characteristic (ROC) analysis was undertaken to determine the ability of wire deflection to predict stability, comparing the measurements from stable situations with those from unstable or indeterminate situations. Statistical analysis was undertaken using Analyse-it for Microsoft Excel, and significance was assumed at the $p<0.05$ level.

\section{Results}

Fourteen patients were recruited to the study. Nine patients were treated for acute fractures, three for deformity correction and two for salvage of fracture-related infections. Ten patients were treated with llizarov ring fixators and four with hexapod fixators. Half the patients had all-wire fixation and half had hybrid constructs, utilising a mixture of wires and half-pins.

A total of 31 deflection measurements were taken from 14 patients. Fourteen of these observations were from patients with hybrid fixation, the remainder being from patients with all-wire fixators. Following clinician assessment of the clinical situation at each time point, 10 were designated as stable, 7 as indeterminate and 14 as unstable. Ten patients had measurements taken at more than one time point.

Deflection measurement results are shown in Figure 2. The median deflection in the stable group was 0.45 microns $/ \mathrm{kg} / \mathrm{m}$ (IQR $0.22-2.31$, range $0.19-11.27$ ). There was a 10 -fold increase in the median wire deflection measured in the indeterminate group at $4.79 \mathrm{microns} / \mathrm{kg} / \mathrm{m}$ (IQR 3.03-7.30, range 2.37-15.75) and a 15-fold increase in the unstable group at 6.59 (IQR 2.72-13.11, range 0.86-56.40). This difference was statistically significant (WMW test $p=0.0049$ ). Subgroup analysis, controlling for multiple comparisons, revealed that the difference between stable and unstable and stable and indeterminate remained statistically significant $(p=0.008$ and $p=0.03$, respectively) but that the difference between the unstable and indeterminate groups did not $(p=0.93)$.

When considering the patients with multiple measurements at different time points, a clear pattern of decreasing wire deflection over time was observed (Fig. 3A). Examining only those patients in whom an indeterminate and stable measurement was available reveals a similar pattern (Fig. 3B).
Regarding frame construction, taking the data as a whole, median deflection measurements in hybrid frames were larger than in the all-wire frames, though this did not reach statistical significance ( 7.35 microns $/ \mathrm{kg} / \mathrm{m}$ (IQR 2.24-13.53, range 0.20-56.40) vs 2.75 microns $/ \mathrm{kg} / \mathrm{m}$ (IQR 1.12-5.11, range 0.19-11.27), WMW test $p=0.08$ ). When considering only frames in stable or unstable situations in this regard, this difference is much more apparent for measurements taken in an unstable situation (Figs 4A and B). Discrimination between stable and unstable situations was retained when considering only frames of hybrid or all-wire construction, again approaching but not reaching statistical significance (Figs 5A and B). For these analyses, with reduced numbers in the subgroups, statistical significance was approached but not reached.

ROC curve analysis revealed that wire deflection measured in this manner was able to predict clinical stability when comparing stable to indeterminate or unstable situations (AUC $0.87,95 \% \mathrm{Cl}$ $0.70-1.03, p<0.0001)$. A deflection cut-off of $1 \mathrm{micron} / \mathrm{kg} / \mathrm{m}$ was $100 \%$ specific and $60 \%$ sensitive in this set of results. Raising this threshold to 2.3 resulted in $80 \%$ sensitivity and $91 \%$ specificity. This is shown in Figures $6 \mathrm{~A}$ and $\mathrm{B}$.

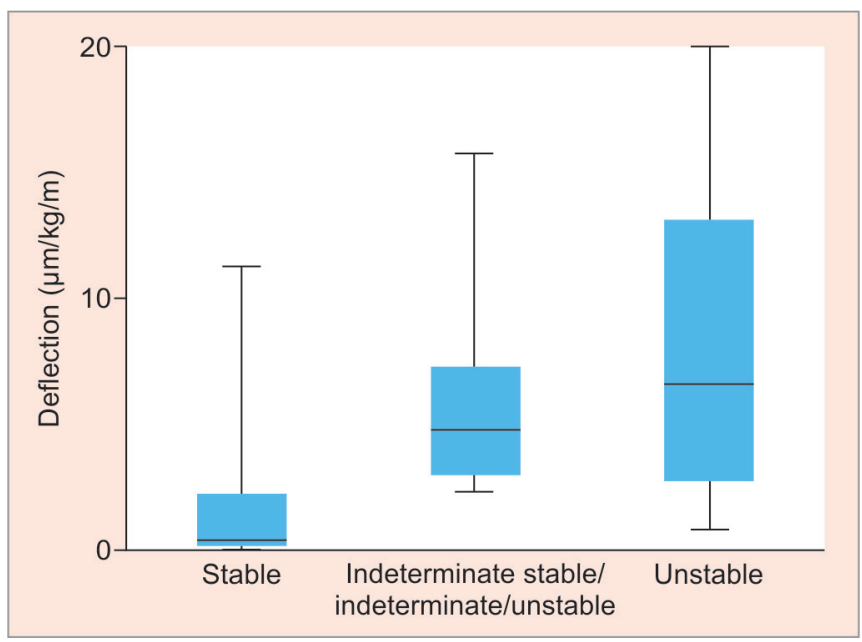

Fig. 2: Wire deflection on loading in stable, indeterminate and unstable situations. Relationship statistically significant (KW test $p=0.005$, SDCF test for multiple comparisons significant for unstable vs stable $(p=0.008)$ and indeterminate vs stable $(p=0.030))$
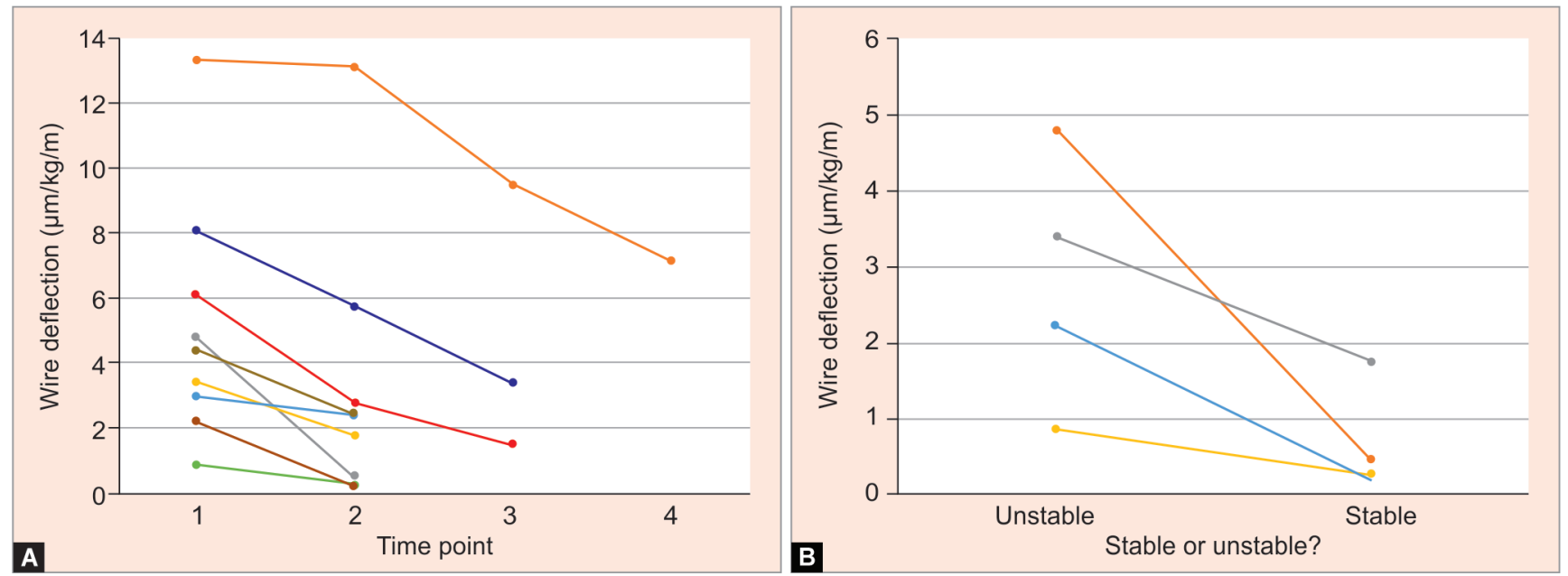

Figs $3 \mathrm{~A}$ and B: Wire deflection on loading in patients with multiple measurements over time-(A) All patients; (B) In patients with a measurement taken in stable and unstable situations 

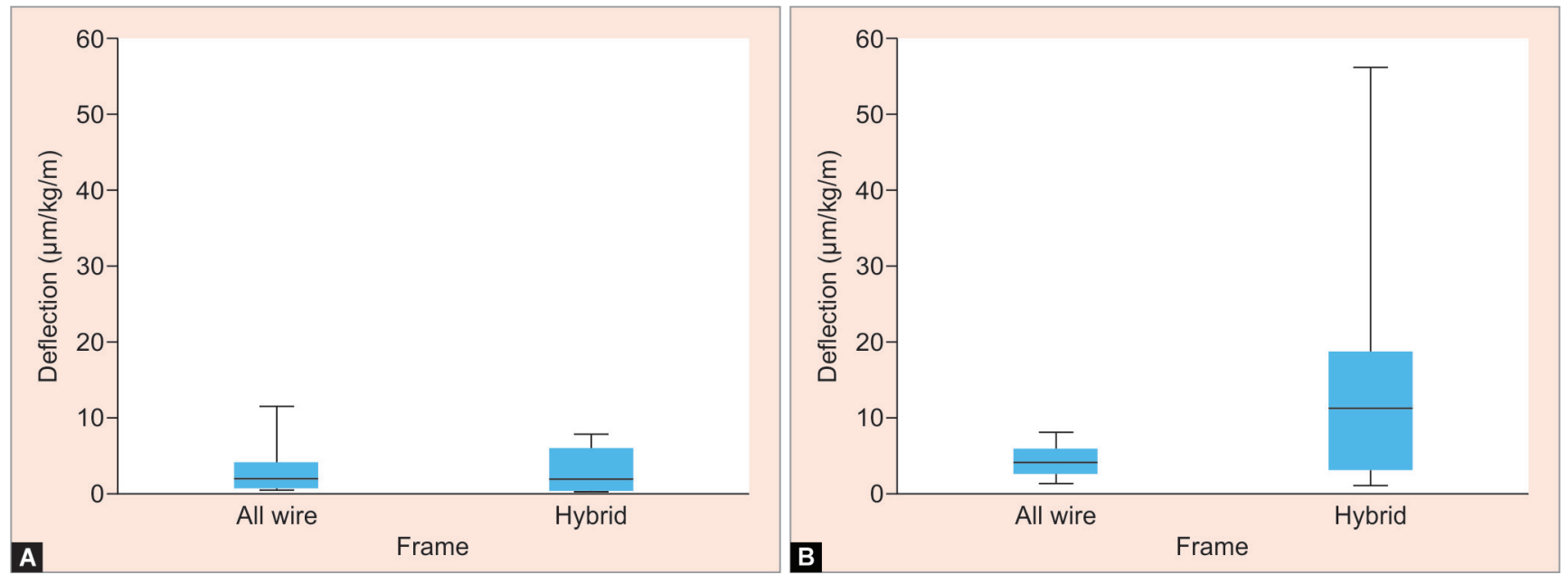

Figs 4A and B:Wire deflection on loading comparing all-wire and hybrid frames in (A) Stable situations (KW test $p=0.877)$; (B) Unstable situations (KW test $p=0.091)$
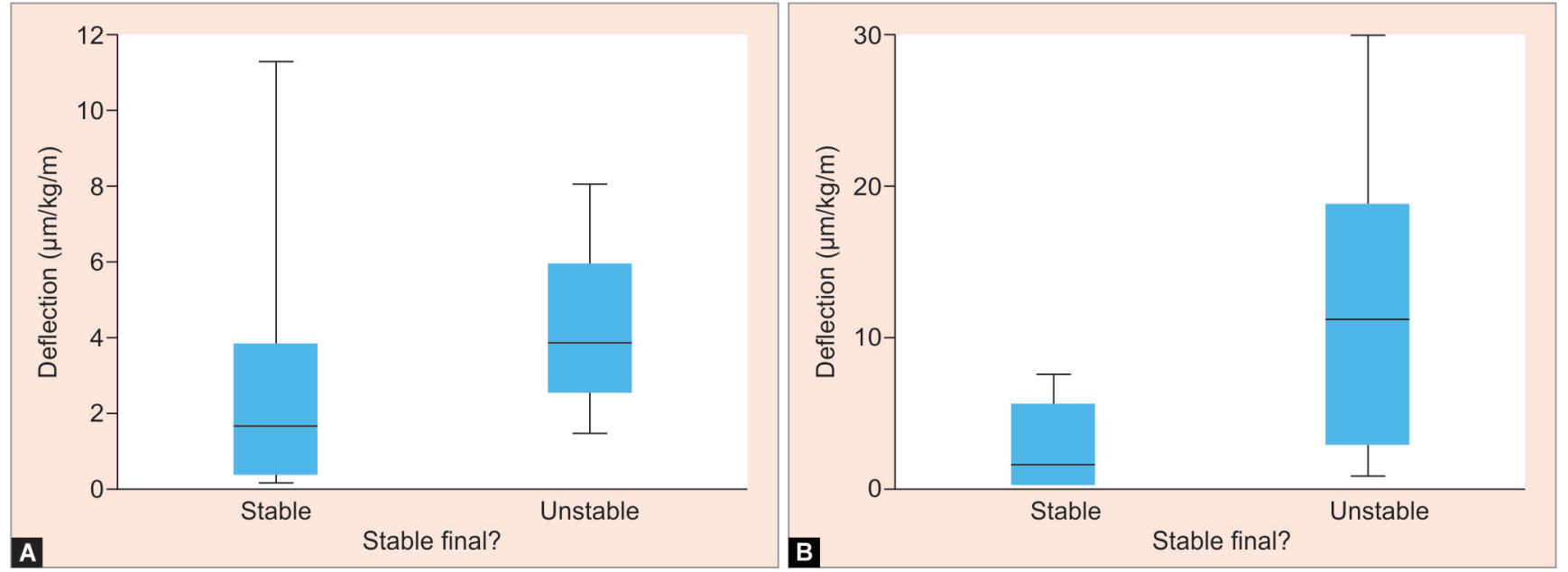

Figs $5 \mathrm{~A}$ and B: Wire deflection on loading comparing stable and unstable situations in (A) All-wire frames (KW test $p=0.101)$; (B) Hybrid frames (KW test $p=0.066)$
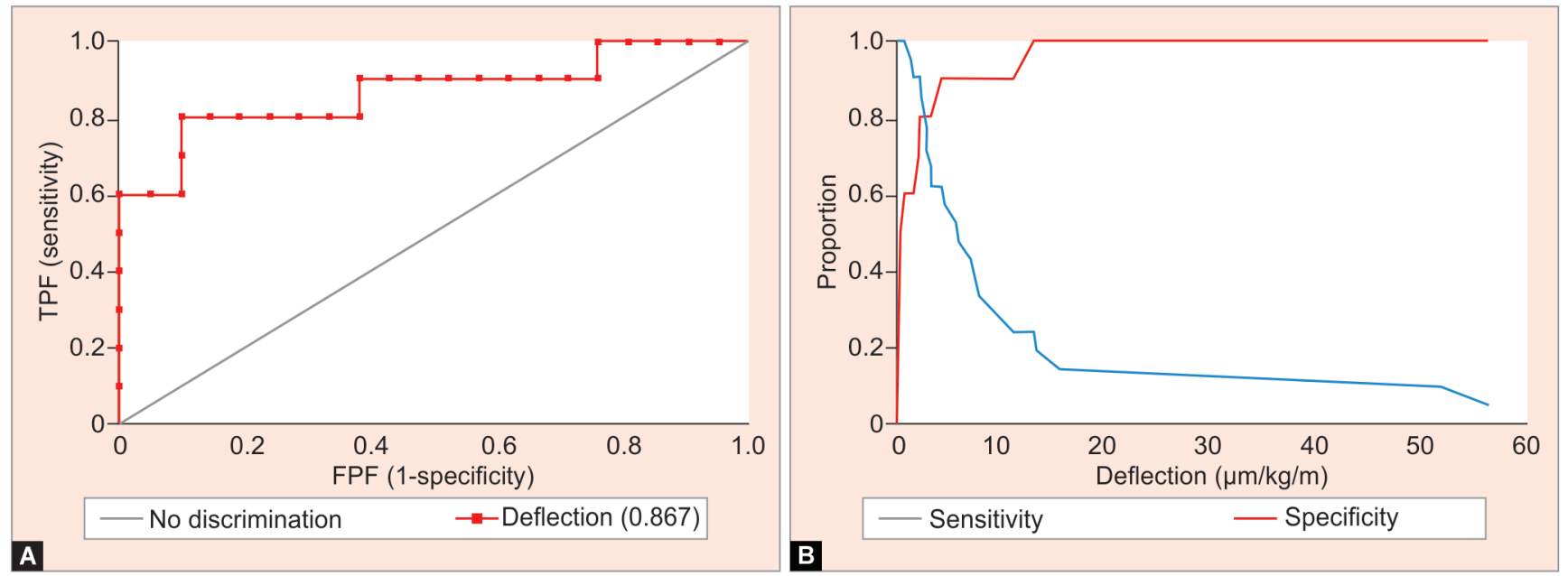

Figs 6A and B: Receiver-operator characteristic (ROC) analysis of wire deflection to predict stability in the study group: (A) ROC curve; (B) Decision threshold analysis 


\section{Discussion}

This study demonstrates a relationship between wire deflection on weight-bearing and progression to union in patients treated with llizarov frames. There was a significant difference between measurements where clinical assessment determined that the fracture was likely to be mechanically stable and where it was deemed unstable. This suggests that wire deflection measurements taken in this manner likely correlate with progress to bony union. All patients with data captured at multiple time points had a decreasing trend of wire deflection per kg over time. Although wire deflection has not previously been assessed in a clinical setting, this corresponds with studies demonstrating increasing rigidity of the external fixator construct as bone progresses to union despite an expected loss of rigidity in the fixator construct over time due to cyclical loading. ${ }^{8,9,12}$

A potential issue with measuring wire deflection as described in this study is loss of wire tension due to slippage of the fixation bolts and plastic deformation of the wires over time..$^{13-15}$ In this study, patients who had measurements taken at different time points demonstrated progressively decreasing wire deflection in later measurements. One explanation for this is that as the fracture site becomes more rigid due to callus maturation, force transmission becomes predominantly intrinsic. If load is increasingly being transferred via the patient's own tissues rather than the frame, the rigidity of the circular fixator will have less influence on overall construct stability. Decreasing rigidity of the frame construct due to loss of wire tension in this situation would not therefore be reflected by increasing wire deflection if union is occurring. In fractures which were slow to or failed to unite, load transfer should remain via the fixator meaning that larger wire deflection should be observed which may even increase, rather than decrease over time. This was not, however, observed in any of the cases presented here. Conversely, loss of wire tension early in treatment may predispose to slower union due to decreased overall fixator rigidity. The ability to observe this phenomenon more closely using the techniques described here is potentially of interest for further studies and may ultimately be of use in clinical practice.

Unfortunately, when comparing all-wire and hybrid frames, the subgroups become smaller, and therefore, statistical analysis did not reach significance at the $p<0.05$ level. Nevertheless, the trends observed are potentially of interest. The fact that deflection between constructs in situations judged stable (Fig. 4A) is similar is perhaps expected, given the assumption that the majority of load transfer should be intrinsic in this situation. The frame should have little influence on overall mechanics if the bone has united. In contrast, the results in unstable situations appear very different (Fig. 4B), with larger deflections observed in the hybrid fixator group. This suggests that the hybrid constructs in this study were less rigid than the all-wire fixators at the time of measurement. The results comparing wire deflection in unstable and stable situations in all-wire and hybrid constructs (Figs 5A and B) appear to show that wire deflection is able to differentiate between stable and unstable situations when assessing all-wire and hybrid constructs. These results need to be interpreted with caution, and the groups are small and the analysis non-significant. The patients were very heterogeneous meaning that the groups were not directly comparable and differences other than the use of half-pins in the construct may have influenced the deflection in each group. These observations would require further investigation in a larger group of patients.
The main limitation of this study was the low number of patients and heterogeneity of indications for frame management and of the constructs themselves. We were unable to obtain large numbers of serial measurements in individual patients, and direct comparison between groups was not possible. Also, as there is no absolutely reliable method for determining union, comparison of the deflection results was with clinical estimation of the stability of the fracture. This was undertaken by one researcher and therefore is potentially not reproducible. Previous methods of assessing bone union using mechanical means have shown indirect measurements of stability to correlate with union. ${ }^{16}$ However, these were not practical to use clinically. The investigators in this study required minimal training to effectively use this method. The risks involved in this method are low with no adjustment of the frame necessary. Measurements can be taken as part of a standard clinic visit. The components are non-toxic and can easily be cleaned with an alcohol-based wipe in clinical practice and reused. Various technical problems were encountered when using the device which would potentially limit its clinical utility in its current form. These consisted of stable fixation of the device onto the construct and adequate clearance between frame and patient to allow for measurements. We now plan to develop a specifically designed tool to overcome these technical issues following the promising results of this method.

The technique described here shows the potential for measurement of wire deflection, or indeed other indicators of force transfer to the frame, to contribute to the assessment of patients undergoing management with circular frames. Serial measurements in the same patient appear likely to have the most potential rather than comparison with an absolute value, as this would accommodate for differences in frame design which would remain constant between measurements. It seems likely that observed force transfer would decrease with time and it should be possible with experience to use this information to judge union. If it were observed that wire deflection or force transfer suddenly increased, this might suggest a problem with the frame such as wire loosening or breakage which might require intervention. It might be that a device could be built into the frame which facilitated recording these parameters by the patient at home or facilitated constant monitoring. This might contribute to more bespoke arrangements for follow-up, triggering outpatient visits with radiographs at appropriate points based on mechanics rather than at arbitrary time points. The practical problems encountered resulted in the measuring device being more difficult and time-consuming to apply in clinic than anticipated. It was therefore decided to terminate the pilot study with a view to constructing a more user-friendly device based upon this work, for use in a further larger clinical study exploring these concepts further.

\section{Conclusion}

In this set of observations, wire deflection was significantly associated with clinically and radiologically determined stability. Though various practical limitations were encountered in using the prototype measurement device, this proof-of-concept study supports further development of this approach. The research group plan to develop a smaller, more reliable device for further clinical testing in a larger group of patients. 


\section{References}

1. Babatunde OM, Fragomen A, Rozbruch R. Noninvasive quantitative assessment of bone healing after distraction osteogenesis. HSS J 2007;6(1):71-78. DOI: 10.1007/s11420-009-9130-y.

2. Corrales $L A$, Morshed $S$, Bhandari $M$, et al. Variability in the assessment of fracture-healing in orthopaedic trauma studies. J Bone Joint Surg Am 2008;90A(9):1862-1868. DOI: 10.2106/JBJS.G.01580.

3. Richardson J.B., Hardy J.R.W. (2000) The Measurement of Fracture Healing. In: De Bastiani G., Apley A.G., Goldberg A. (eds) Orthofix External Fixation in Trauma and Orthopaedics. Springer, London. DOI: 10.1007/978-1-4471-0691-3_5.

4. Watson MA, Mathias KJ, Maffulli N. External ring fixators: an overview. Proc Inst Mech Eng H 2000;214(5):459-470. DOI: 10.1243/0954411001535480.

5. Bail HJ, Kolbeck S, Krummrey G, et al. Ultrasound can predict regenerate stiffness in distraction osteogenesis. Clin Orthop Relat Res 2002;(404):362-367. DOI: 10.1097/00003086-200211000-00053.

6. Eyres KS, Bell MJ, Kanis JA. Methods of assessing new bone formation during limb lengthening. Ultrasonography, dual energy X-ray absorptiometry and radiography compared. J Bone Joint $\mathrm{Br} 1993$; 75-B(3):358-364. DOI: 10.1302/0301-620X.75B3.8496200.

7. Markel $M, C h a o E$. Noninvasive monitoring techniques for quantitative description of callus mineral content and mechanical properties. Clin Orthop Relat Res 1993;(293):37-45. PMID: 8339505.

8. Cunningham JL, Kenwright J, Kershaw CJ. Biomechanical measurement of fracture healing. J Med Eng Technol 1990;14(3): 92-101. DOI: 10.3109/03091909009015420.
9. Richards J. Stiffness in healing fractures. Crit Rev Biomed Eng 1987;15(2):145-185. PMID: 3319415.

10. Gessmann J, Baecker H, Jettkant B, et al. Direct and indirect loading of the llizarov external fixator: the effect on the interfragmentary movements and compressive loads. Strategies Trauma Limb Reconstr 2011;6(1):27-31. DOI: 10.1007/s11751-011-0103-6.

11. Lineham B, Stewart T, Harwood P. Measurement of wire deflection on loading may indicate union in llizarov constructs, an in vitro model. Strategies Trauma Limb Reconstr 2018;13(2):75-80. DOI: 10.1007/ s11751-018-0306-1.

12. Kristiansen B, Borgwardt A. Fracture healing monitored with strain-gauges-external fixation of 7 humeral neck fractures. Acta Orthop Scand 1992;63(6):612-614. DOI: 10.1080/17453679209169719.

13. Aquarius R, Van Kampen A, Verdonschot N. Rapid pre-tension loss in the llizarov external fixator: an in vitro study. Acta Orthop 2007;78(5):654-660. DOI: 10.1080/17453670710014356.

14. Gessmann J, Jettkant B, Königshausen M, et al. Improved wire stiffness with modified connection bolts in llizarov external frames: a biomechanical study. Acta Bioeng Biomech 2012;14(4):15-21. PMID: 23394181.

15. La Russa V, Skallerud B, Klaksvik J, et al. Reduction in wire tension caused by dynamic loading. An experimental llizarov frame study. J Biomech 2011;44(8):1454-1458. DOI: 10.1016/ j.jbiomech.2011.03.018.

16. Claes LE, Cunningham JL. Monitoring the mechanical properties of healing bone. Clin Orthop Relat Res 2009;467(8):1964-1971. DOI: 10.1007/s11999-009-0752-7. 